Draft Version November 9, 2018

Preprint typeset using $\mathrm{I}_{\mathrm{A}}^{\mathrm{T}} \mathrm{EX}$ style emulateapj v. 03/07/07

\title{
COMPOSITION OF COSMIC RAYS ACCELERATED IN ACTIVE GALACTIC NUCLEI
}

\author{
E.G.BEREZHKO ${ }^{1}$ \\ Draft version November 9, 2018
}

\begin{abstract}
The composition of the overall spectrum of cosmic rays (CRs) is studied under the assumption that ultra high energy CRs above the energy $10^{17} \mathrm{eV}$ are produced at the shock created by the expanding cocoons around active galactic nuclei (AGNs). It is shown that the expected CR composition is characterised by two peaks in the energy dependence of the mean CR atomic number $\langle A(\epsilon)\rangle$. The first one at the energy $\epsilon \approx 10^{17} \mathrm{eV}$ corresponds to the very end of the Galactic CR component, produced in supernova remnants (SNRs). It is followed by a sharp decrease of $\langle A(\epsilon)\rangle$ within the energy interval from $10^{17}$ to $10^{18} \mathrm{eV}$. This is a signature of the transition from Galactic to extragalactic CRs. The second peak, with $<\ln A>\approx 2$, at energy $\epsilon \approx 10^{19} \mathrm{eV}$, expected at the beginning of the GZK cutoff, is the signature of the CR production by the nonrelativistic cocoon shocks. The calculated CR composition is consistent with the existing data. The alternative scenario, which suggests reacceleration increasing the energy of CRs produced in SNRs by a factor of 30, is also examined.

Subject headings: acceleration of particles — cosmic rays — galaxies: active - shock waves - supernova remnants
\end{abstract}

\section{INTRODUCTION}

The overall origin of cosmic rays (CR), in particular at ultra high energies, above $10^{18} \mathrm{eV}$, is still an unresolved problem in astrophysics (e.g. Nagano \& Watson 2000; Stanev 2004; Cronin 2005; Inoue 2008). Understanding this origin requires the determination of the astrophysical objects, that are the CR sources, and of the appropriate acceleration processes, that form the CR spectrum in these objects. During the last several years considerable progress has been achieved in this field, both experimentally and theoretically. Recently the sharp steepening of the CR spectrum above $3 \times 10^{19} \mathrm{eV}$ was established in the HiRes (Bergman et al. 2007) and Auger (Yamamoto et al. 2007) experiments. It presumably corresponds to the so-called Greizen-Zatsepin-Kuzmin (GZK) cutoff, caused by energy losses of the CRs in their interactions with the microwave background radiation. This is evidence that the highest energy part of the CR spectrum is of extragalactic origin. It was also recently demonstrated (Berezhko \& Völk 2007) that the CRs with energies up to $\epsilon \sim 10^{17} \mathrm{eV}$ can be produced in supernova remnants (SNRs) as a result of diffusive shock acceleration (Krvmskv 1977; Bell 1978), and that the observed CR energy spectrum can be well represented by two components: the first, dominant up to $10^{17} \mathrm{eV}$, consists of CRs produced in Galactic SNRs; the second, dominant at energies above $10^{18} \mathrm{eV}$, is of extragalactic origin. The latter component can be produced at the shock that is formed by the expanding cocoon around active galactic nuclei (AGNs): above the energy $10^{18} \mathrm{eV}$ the overall energy spectrum of CRs, produced during the AGN evolution and released into intergalactic space, has an appropriate power law form, which extends at least up to the energy $\epsilon_{\max } \sim 10^{20} \mathrm{eV}$, well above the GZK cutoff (Berezhko 2008). This is the "dip scenario"

\footnotetext{
Electronic address: berezhko@ikfia.ysn.ru

${ }^{1}$ Yu.G. Shafer Institute of Cosmophysical Research and Aeronomy, 31 Lenin Ave., 677980 Yakutsk, Russia
}

of the overall CR spectrum formation (Berezinsky et al. 2006). Within the alternative "ankle scenario", extragalactic CRs are expected to dominate only above the energy $10^{19} \mathrm{eV}$ (Berezinsky et al. 2006). It was noted (Berezhko \& Völk 2007; Berezhko 2008), that the composition of CRs at $\epsilon>10^{17} \mathrm{eV}$ is expected to be very different in these two scenarios. Therefore the study of CR composition can in principle provide the signature of the acceleration mechanism which creates the spectrum of extragalactic CR component.

Here we present the calculations of the expected composition of CRs, accelerated by cocoon shocks and demonstrate that it has well pronounced peculiarities, which can be considered as a signature of ultra high energy CR production by nonrelativistic shocks. The calculated composition is compared with the composition expected within the alternative scenario and with the existing data. The alternative scenario, which suggests reacceleration increasing the energy of CRs produced in SNRs by a factor of 30 , is also examined.

\section{RESULTS AND DISCUSSION}

The composition of CRs is determined if we know not only their all-particle spectrum,

$$
J(\epsilon)=\sum_{A} J_{\mathrm{A}}(\epsilon),
$$

that is the differential intensity $J(\epsilon)$ with respect to particle energy $\epsilon$, but also the spectra $J_{\mathrm{A}}(\epsilon)$ of all relevant elements with atomic number $A$. Since the shockaccelerated CRs come originally from the thermal background plasma, one has to expect that the flux of each $\mathrm{CR}$ element in/near the source, is proportional to the number density of this element $N_{\mathrm{A}}$ in the background plasma: $J_{\mathrm{A}}^{\mathrm{s}}(\epsilon) \propto N_{\mathrm{A}}$. Therefore it is useful to represent the spectrum of each CR element in the form

$$
J_{\mathrm{A}}^{\mathrm{s}}(\epsilon)=e_{\mathrm{A}}(\epsilon) a_{\mathrm{A}} J_{\mathrm{H}}^{\mathrm{s}}(\epsilon),
$$

where $a_{\mathrm{A}}=N_{\mathrm{A}} / N_{\mathrm{H}}$ is the abundance of element $A$ relative to the hydrogen abundance and $e_{\mathrm{A}}(\epsilon)$ is an en- 
richment factor which describes the preferential production of element $A$ relative to the production of protons (marked by the subscript $\mathrm{H}$ ).

Direct measurements of CR fluxes at energies $\epsilon<$ $10^{14} \mathrm{eV}$ show that the factor $e_{\mathrm{A}}>1$ considerably exceeds unity and that it progressively increases with the increase of $A$ (e.g. Berezhko \& Ksenofontov 1999). This means a considerable enrichment of CRs in heavy elements compared with the interstellar abundance. Note that at these relatively low energies, where direct determination of $\mathrm{CR}$ particle parameters is possible, the fluxes $J_{\mathrm{A}}$ and $J_{\mathrm{H}}$ are usually compared at the same energy per nucleon, that is for $\epsilon_{\mathrm{A}}=A \epsilon_{\mathrm{H}}$. Since it is impossible to determine the mass of individual $\mathrm{CR}$ particles at ultra high energies, the particle energy instead of the energy per nucleon is the most appropriate variable for the analysis of CR spectrum and composition in this case.

The observed CR composition at $\epsilon<10^{17} \mathrm{eV}$ is well consistent with the scenario in which Galactic CR component is produced by supernova shocks (Berezhko \& Völk 2007). The enrichment of shockaccelerated CRs by heavy elements is due to a more effective injection and subsequent acceleration of heavy ions (see Berezhko \& Ksenofontov 1999, for the details).

The spectra of ultrahigh energy CRs produced by nonrelativistic cocoon shocks can be represented in the form (Berezhko 2008)

$$
J_{\mathrm{A}}^{\mathrm{s}}(\epsilon)=C_{\mathrm{A}} \epsilon^{-\gamma},
$$

where $\gamma \approx 2.6$ and the values of parameters $C_{\mathrm{A}} \propto e_{\mathrm{A}} N_{\mathrm{A}}$ are determined by the background medium's composition $N_{\mathrm{A}}$ and by the enrichment factors $e_{\mathrm{A}}$. Since the injection/acceleration of CRs at cocoon and supernova shocks are expected to be very similar, the values of the enrichment factors $e_{\mathrm{A}}$ in the former case can be extracted from the experimentally measured fluxes of the Galactic CR component $J_{\mathrm{A}}(\epsilon)$ at some fixed energy, say $\epsilon=1 \mathrm{TeV}$ (see e.g. Berezhko \& Völk 2007), according to the expression

$$
e_{\mathrm{A}}=J_{\mathrm{A}}(1 \mathrm{TeV}) / J_{\mathrm{H}}(1 \mathrm{TeV})\left(N_{\mathrm{H}} / N_{\mathrm{A}}\right) \odot .
$$

Here $\left(N_{\mathrm{A}} / N_{\mathrm{H}}\right)_{\odot}$ is the solar system relative abundance of elements with atomic number $A$.

In order to calculate the spectra of extragalactic CRs inside the Galaxy one needs to take into account the modification of the CR spectra due to their interaction with the cosmic microwave background (CMB) and due to their modulation in the Galactic wind. This can be done by representing the observed extragalactic CR spectrum in the form

$$
J_{\mathrm{A}}(\epsilon)=P_{1} P_{\mathrm{h}} J_{\mathrm{A}}^{\mathrm{s}}(\epsilon),
$$

where factors $P_{1}$ and $P_{\mathrm{h}}$ describe $\mathrm{CR}$ flux modification due to Galactic wind and $\mathrm{CMB}$ respectively. Since the diffusive mobility of the CRs is inversely proportional to the particle charge number $Z$, the first factor can be represented in the form

$$
P_{1}=\exp \left(-Z \epsilon_{1} / \epsilon\right),
$$

where $\epsilon_{1} \sim 10^{17} \mathrm{eV}$ (Berezhko \& Völk 2007). This expression shows that at a given energy the lighter CR species can more easily penetrate the inner Galaxy from outside. Due to this factor the observed extragalactic CR component becomes progressively heavier with increasing energy.

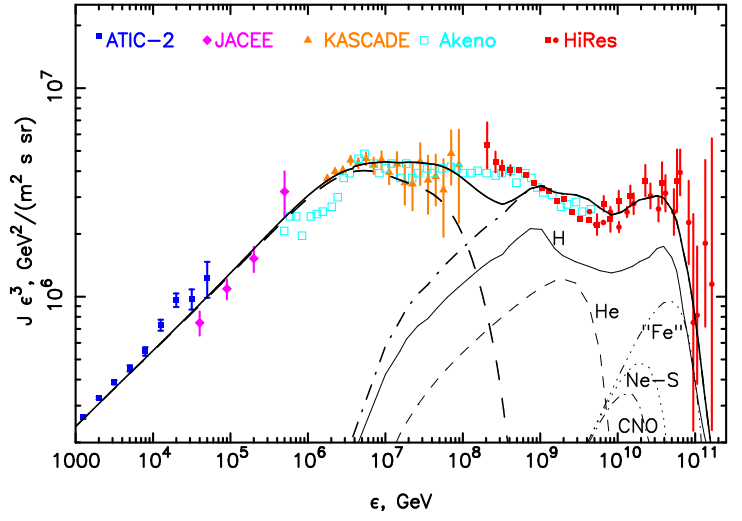

FIG. 1.- Overall CR intensity as a function of energy (thick solid line), Galactic CR component produced in SNRs (thick dashed line) and extragalactic component produced in the IGM (thick dash-dotted line). The spectra of different extragalactic components are shown by thin lines. Experimental data obtained in the ATIC-2 (Panov et al. 2006), JACEE(Asakimori et al.|2003), KASCADE (Antoni et al.2005), Akeno (Takeda et al. 2003), and HiRes (Bergman et al.|2007) experiments are shown as well.

To describe the effects of CRs interacting with the CMB radiation during their propagation from extragalactic sources to the observer - pion and pair production, CR nuclei photodesintegration and adiabatic cooling - we use a simple analytical form of the factor $P_{\mathrm{h}}$

$$
P_{\mathrm{h}}=\exp \left[-\left(A \epsilon / \epsilon_{\mathrm{h}}\right)^{2}\right] \text {. }
$$

At $\epsilon_{\mathrm{h}}=1.3 \times 10^{18} \mathrm{eV}$ for the case of helium and iron nuclei it satisfactorily fits the results of detailed calculations which consistently describes all these effects (Berezinsky et al. 2006). In the case of protons, instead of the modification factor $P_{\mathrm{h}}$ we directly use their modified spectrum calculated by Berezinsky et al. (2006).

As is clear from expression (7), the interaction of CR nuclei with the CMB radiation produces a sharp cutoff in their spectra at the energy, which is proportional to the nuclear mass. Due to this fact CRs are expected to become progressively heavier at energies above $10^{18} \mathrm{eV}$.

The cocoon shock propagates initially through the interstellar medium (ISM) of the host galaxy and later through the intergalactic medium (IGM). The IGM is less abundant in heavy elements than the ISM: the median metallicity of the IGM is $\left(N_{\mathrm{A}} / N_{\mathrm{H}}\right)=$ $0.2\left(N_{\mathrm{A}} / N_{\mathrm{H}}\right) \odot$ for all relevant elements heavier than helium (e.g. Cen \& Ostriker 2006). Since the maximal CR energy produced by the expanding cocoon shock decreases with time (Berezhko 2008), the local metallicity at the shock front changes from the ISM value $\left(N_{\mathrm{A}} / N_{\mathrm{H}}\right) \approx\left(N_{\mathrm{A}} / N_{\mathrm{H}}\right) \odot$ at early phases, when the shock produces CRs up to $\epsilon_{\max } \sim Z \times 10^{20} \mathrm{eV}$, to the IGM value at late phases, when $\epsilon_{\max } \sim Z \times 10^{18} \mathrm{eV}$. To describe this effect we uses a metallicity $\left(N_{\mathrm{A}} / N_{\mathrm{H}}\right)=$ $0.2\left[\epsilon /\left(Z \times 10^{18} \mathrm{eV}\right)\right]^{0.35}\left(N_{\mathrm{A}} / N_{\mathrm{H}}\right) \odot$. For helium we use the cosmological value $N_{\mathrm{He}}=0.08 N_{\mathrm{H}}$ (e.g. Berezinsky et al. 2006). Using these number densities one can calculate the relative values of the coefficients $C_{\mathrm{A}} / C_{\mathrm{H}}$ for all elements except protons. The proton coefficient $C_{\mathrm{H}}$ depends on a number of physical factors such as the IGM hydrogen number density $N_{\mathrm{H}}$ and the power and spatial distribution of nearby AGNs. Its value is determined by the fit of the expected all-particle CR flux $J(\epsilon)$ to the existing measurements of the $\mathrm{CR}$ intensity at energies $\epsilon>10^{18} \mathrm{eV}$. 


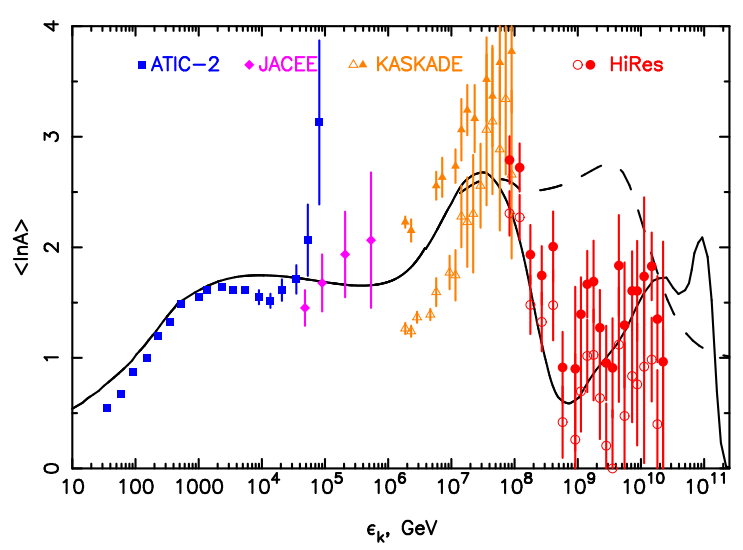

FIG. 2.- Mean logarithm of the CR nucleus atomic number as a function of energy. Solid and dashed lines correspond to the dip and ankle scenarios respectively. Experimental data obtained in the ATIC-2, JACEE, KASCADE (QGSJET and SYBYLL; Hörande] 2005), and HiRes (QGSJET and SYBYLL; Abbasi et al. 2005) experiments are shown.

Secondary protons appearing as a result of photodesintegration of helium nuclei are also included in an approximate way: it is assumed that the secondary protons (whose total number equals four times the number of helium nuclei in the source spectrum with energy above $2 \times 10^{18} \mathrm{eV}$ ) have the normal energy distribution around $\epsilon_{0}=5 \times 10^{17} \mathrm{eV}$ within the interval $\Delta \epsilon=\epsilon_{0}$.

In Fig.1 we present the all-particle spectrum which includes two components: CRs produced in SNRs (Berezhko \& Völk 2007) and extragalactic CRs, which consist of protons $(\mathrm{H})$, helium $(\mathrm{He})$ and three groups of heavier nuclei, produced in AGNs. Note, that the dip at energy $\epsilon \approx 10^{19} \mathrm{eV}$ is still clearly seen even though it is less pronounced compared with the case of pure proton composition. The calculated overall CR spectrum is in a satisfactory way consistent with the experimental data, except in the energy interval $10^{17}<\epsilon<10^{18} \mathrm{eV}$ in the transition region, where, contrary to the experiment, the calculated spectrum has a small dip. Taking into account the existing experimental uncertainties at these energies it is not clear whether this discrepancy is a serious problem for the model or not. Note, that the size and the shape of this peculiarity is very sensitive to the details of the galactic CR high energy cutoff and to the extragalactic low energy spectrum part. Therefore it is hard to predict it quantitatively. At the same time the overall spectrum, which is a mixture of two independent components, should have such peculiarity within the transition region. If the actual $\mathrm{CR}$ spectrum is indeed so uniform within the energy range $10^{17}<\epsilon<10^{18} \mathrm{eV}$, as it looks according to the Akeno data, then it should be considered as a serious problem for the dip scenario.

The mean logarithm of $\mathrm{CR}$ atomic number is represented in Fig.2 as a function of energy. As already qualitatively predicted (Berezhko 2008), the CR mass energy dependence $<\ln A(\epsilon)>$ has two peaks. The first one at the energy $\epsilon \approx 10^{17} \mathrm{eV}$ corresponds to the very end of the galactic CR component (Berezhko \& Völk 2007), whereas the second, at the energy $\epsilon \approx 10^{19} \mathrm{eV}$, is at the beginning of the GZK cutoff. Note that at high energies $\epsilon>10^{15} \mathrm{eV}$ information about $\mathrm{CR}$ composition is obtained from the mean values of the shower maxima $X_{\max }$ (usually measured in $\mathrm{g} / \mathrm{cm}^{2}$ ), determined by the ground based detectors. Knowing the average depth of the shower maximum for protons, $X_{\max }^{\mathrm{H}}$, and for iron nuclei, $X_{\max }^{\mathrm{Fe}}$, from simulations, the mean logarithmic mass can be estimated from the measured $X_{\max }$ according to the relation (e.g. Hörandel 2005)

$$
<\ln A>=\left(X_{\max }-X_{\max }^{\mathrm{H}}\right) /\left(X_{\max }^{\mathrm{Fe}}-X_{\max }^{\mathrm{H}}\right)<\ln 56>\text {. }
$$

This conversion requires the choice of a particle interaction model. Here we use the values of $X_{\max }$, measured in the HiRes experiment (Abbasi et al. 2005), and the QGSJET and SYBYLL models to determine $<\ln A>$. It is seen from Fig. 2 that the HiRes data are very well consistent with the expected sharp decrease of $\langle\ln A\rangle$ within the energy interval $10^{17}-10^{18} \mathrm{eV}$. At higher energies, between $10^{18}$ and $10^{19} \mathrm{eV}$, the experimental values of $\langle\ln A(\epsilon)>$ have a quite irregular behavior. Nevertheless, the experiment reveals a trend of progressive increase of the mean $\mathrm{CR}$ mass so that the expected peak value $\left\langle\ln A>\approx 1.7\right.$ at the energy $\epsilon \sim 10^{19} \mathrm{eV}$ is consistent with the existing HiRes data. It is also clear from Fig.2, that a more precise experimental determination of the $\mathrm{CR}$ mass composition for $\epsilon>10^{15} \mathrm{eV}$ is required for a more complete conclusion about the $\mathrm{CR}$ origin.

\section{ALTERNATIVE SCENARIO}

The alternative scenario for the overall $\mathrm{CR}$ spectrum is the "ankle scenario" (Berezinsky et al. 2006). In this case the extragalactic source spectrum, as compared with the dip-scenario, is assumed to be much harder $J_{\mathrm{A}}^{\mathrm{s}} \propto \epsilon^{-2}$ so that it becomes dominant above an energy of $\epsilon=10^{19} \mathrm{eV}$ (e.g. Berezinsky et al. 2006). Therefore, to fit the observed overall CR spectrum one needs a third component to fill the gap between the Galactic CR spectrum, produced by SNRs, and the hard extragalactic spectrum. It is hard to believe that this third CR component could be completely independent of the CR component produced in SNRs, since in such a case one should expect some peculiarity in the spectral shape at energy $\epsilon \approx 10^{17} \mathrm{eV}$, where these two components are expected to match. The appropriate solution of this problem is the existence of some kind of reacceleration process which picks up the most energetic CRs from SNRs and substantially increases their energy, resulting in a smooth extension of the first CR component towards the higher energies.

We model the spectra of reaccelerated CRs in the following way, without specifying the reacceleration mechanism. For every element with the nuclear charge number $Z$, as in the dip scenario, we use the spectrum, which coincides with $J_{\mathrm{A}}(\epsilon)$ for $\epsilon<\epsilon_{\max 1}^{\mathrm{Z}}$ and has a form

$$
J_{\mathrm{A}}(\epsilon)=J_{\mathrm{A}}\left(\epsilon_{\max 1}^{\mathrm{Z}}\right)\left(\epsilon / \epsilon_{\max 1}^{\mathrm{Z}}\right)^{-\gamma} \exp \left(-\epsilon / \epsilon_{\max 2}^{\mathrm{Z}}\right)
$$

at $\epsilon>\epsilon_{\max 1}^{\mathrm{Z}}$. Here $\epsilon_{\max 1}^{\mathrm{Z}}$ is the minimum energy of particles involved in reacceleration, and $\epsilon_{\max 2}^{Z}$ is the maximum particle energy achieved during reacceleration. It is natural to assume that these energies scale proportional to the rigidity $\epsilon_{\max }^{\mathrm{Z}}=Z \epsilon_{\max }^{\mathrm{p}}$. Here the superscript $p$ denotes protons. The quantities $\epsilon_{\max 1}^{\mathrm{p}}, \epsilon_{\max 2}^{\mathrm{p}}$ and $\gamma$ are treated as free parameters whose values are determined as a result of the best fit. CR acceleration by spiral density shocks in the Galactic Wind (Völk \& Zirakashvili 2004), or acceleration in the vicinity of pulsars (Bell 1992; Berezhko 1994) could play the role of the reacceleration mechanism. 


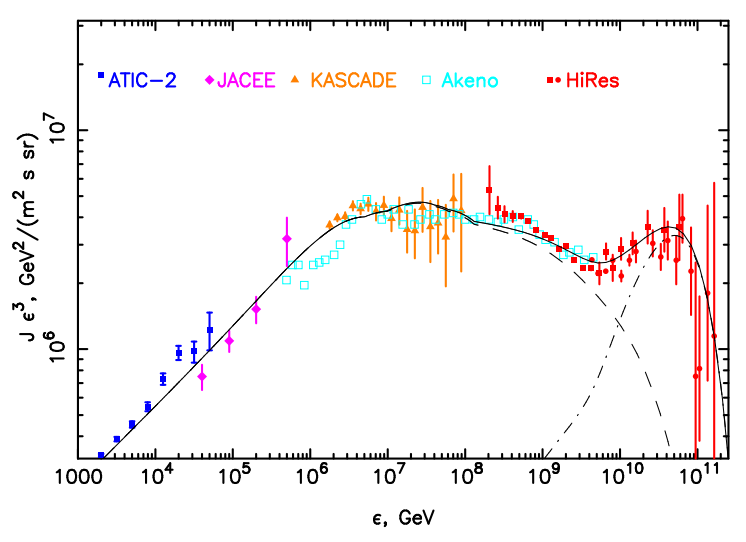

FIG. 3.- The same as in Fig.1, but for the ankle scenario. The dashed line represents the Galactic component, which includes CRs produced in SNRs and reaccelerated CRs. The dash-dotted line represents the extragalactic component; it corresponds to the source spectrum $J_{\mathrm{A}}^{\mathrm{s}} \propto \epsilon^{-2}$ (Berezinsky et al. 2006).

We present in Fig. 3 the CR spectrum calculated within the ankle scenario, with the adopted values $\epsilon_{\max 1}^{\mathrm{p}}=$ $5 \times 10^{15} \mathrm{eV}, \epsilon_{\max 2}^{\mathrm{p}}=1.5 \times 10^{17} \mathrm{eV}$ and $\gamma=3$, which provide the best fit to the observed CR spectrum. Following Berezinsky et al. (2006) we use the extragalactic CR source spectrum in the form $J_{\mathrm{A}}^{\mathrm{s}} \propto \epsilon^{-2}$ and assume the composition of these CRs with $\langle A(\epsilon)\rangle=1$.

It is seen that at $\epsilon>10^{17} \mathrm{eV}$ it is well consistent with the data. However, such a well-known peculiarity in CR spectrum as the knee at $\epsilon \approx 3 \times 10^{15} \mathrm{eV}$ is much less pronounced in the theoretical CR spectrum than in the experiment. This may be considered as an indication against the ankle scenario.

As it is seen in Fig.2, the CR composition corresponding to the ankle scenario is considerably heavier at energies $10^{17} \epsilon<10^{19} \mathrm{eV}$ than in the dip scenario and is inconsistent with the HiRes measurements. Note, that CR composition of the reaccelerated CRs is a direct consequence of the heavy $\mathrm{CR}$ composition at the energy $\epsilon>\epsilon_{\max 1}^{Z}$, where the reacceleration is assumed to start, and it is not sensitive to the details of reacceleration process.

Since the expected spectrum of CRs produced in gamma-ray bursts (GRBs) has a form $J^{\mathrm{s}} \propto \epsilon^{-\gamma}$ with $\gamma=2-2.2$ only ankle scenario is possible if GRBs are the the source of extragalactic CR component (see Berezinsky et al. 2006, and references therein). It means that HiRes CR composition is against GRBs as a source of ultra high energy CRs. In addition, as Berezinsky et al. (2006) argued, the energy output of GRBs has a serious problem if they are considered as the main source of the extragalactic CRs. Note also, that based on the data collected at the Auger experiment, correlation between the arrival directions of CRs with energy above $6 \times 10^{19} \mathrm{eV}$ and the position of nearby AGNs has been find (Abraham et al. 2007), that strongly supports AGNs as a prime candidate for the source of ultra high energy CRs.

\section{SUMMARY}

The composition of ultra high energy CRs produced by nonrelativistic cocoon shocks around AGNs is characterised by well pronounced peculiarities which are two peaks in the energy dependence of the mean $\mathrm{CR}$ atomic number $\langle A(\epsilon)>$. The first peak at the energy $\epsilon \approx$ $10^{17} \mathrm{eV}$ corresponds to the very end of the Galactic CR component, produced in SNRs (Berezhko \& Völk 2007), whereas the second, at the energy $\epsilon \approx 10^{19} \mathrm{eV}$, is expected at the beginning of the GZK cutoff. The strong energy dependence of the CR composition within the energy interval from $10^{17}$ to $10^{18} \mathrm{eV}$ is expected as a signature of the transition from heavy Galactic to light extragalactic CRs, whereas the detection of a heavy CR composition at $\epsilon \approx 10^{19} \mathrm{eV}$ has to be considered as the signature of $\mathrm{CR}$ production by the nonrelativistic cocoon shocks.

The existing measurements of CR composition are consistent with the dip scenario with a formation of the CR spectrum in SNRs and AGNs and are inconsistent with the ankle scenario, which includes reacceleration of $\mathrm{CR}$ produced in SNRs as a third CR component. Additional peculiarity in the overall CR spectrum - the dip in the transition region $10^{17}<\epsilon<10^{18} \mathrm{eV}$ - is expected within the dip scenario. Since it is not seen in the existing data it is a real difficulty for the dip scenario. It is therefore clear that a more precise measurements of CR spectrum and composition at energies above $10^{17} \mathrm{eV}$ are needed for a strict determination of CR origin.

I thank H.J. Völk for the helpful discussions. The work was supported by the Russian Foundation for Basic Research (grants 06-02-96008, 07-02-00221)

\section{REFERENCES}

Abbasi, R.U. et al. 2005, ApJ, 622, 910

Abraham, J. (Pier Auger Collaboration) 2007, Science, 318, 938

Antoni, T. et al. 2005, Astropart. Phys., 24, 1

Asakimori, K. et al. 2003, ApJ, 502, 278

Bell, A.R. 1978, MNRAS, 182, 147

Bell, A.R. 1992, Mon. Not. R. Astron. Soc. 257, 493

Berezhko, E.G. 1994, Astronom. Let., 20, 75

Berezhko, E.G. 2008, ApJ, 684, L69

Berezhko, E.G., \& Ksenofontov, L.T. 1999, JETPh, 89, 391

Berezhko, E.G. \& Völk, H.J. 2007, ApJ, 661, L175

Berezinsky, V.S. et al. 2006, Phys. Rev. D, 74, 043005

Bergman, D.R. et al. 2007 Nuclear Phys. B (Proc. Supl.), 165, 19

Cen, R., \& Ostriker, J. 2006, ApJ, 650, 560
Cronin, J. W. 2005, Nucl. Phys. B (Proc. Supl.), 138, 465

Hörandel, J.R. 2005, astro-ph/0508014

Inoue, S. 2008, J. Phys.: Conf. Ser., 120, 062001

Krymsky, G.F. 1977, Soviet Phys. Dokl., 23, 327

Nagano, M., \& Watson, A. A. 2000, Rev. Mod. Phys., 72, 689

Panov, A. D. et al. 2006, astro-ph/0612377

Stanev, T. 2004, High Energy Cosmic Rays (Chichester: Springer)

Takeda, M. et al. 2003, Astropart. Phys. 19, 447

Völk, H.J. \& Zirakashvili, V.N. 2004, A\&A, 417, 807

Yamamoto, T. et al. arXiv:0707.2638v3[astro-ph] (2007) 